\title{
AUTOIMMUNITY
}

\section{Could early biologics prevent disease?}

\section{$c 4$}

a single injection of an anti-CD40L monoclonal antibody

... could prevent the development of pSS, autoimmune thyroiditis and T1DM
New research conducted in mice suggests that early biologic therapy might ameliorate or even prevent autoimmunity. "The ability of prophylactic anti-CD40L to dissolve germinal centres and halt autoimmune humoral responses in our studies suggests that biologics that target CD40/CD40L interaction[s] may have efficacy in diseases that are predated by the appearance of autoantibodies," Mahmoud and colleagues' report asserts. "There is a treatment window where therapeutic intervention may be able to prevent disease development," they go on to explain.

The researchers studied nonobese diabetic (NOD) mice made congenic for the B10.A(4R) strain-derived MHC complex (NOD.H-2h4 mice), which (unlike standard NOD mice) are no longer susceptible to type 1 diabetes mellitus (T1DM). Instead, adult NOD.H-2h4 mice develop a disease that strongly resembles human primary Sjögren syndrome (pSS), characterized by female sex bias, anti-Ro/La autoantibodies (which arise at the time of development of germinal centres in the spleen) and xerostomia associated with salivary gland tertiary lymphoid structures.
Alternatively, upon exposure to sodium iodide, NOD.H-2h4 mice develop a spontaneous autoimmune thyroiditis characterized by autoantibodies and infiltration of the thyroid by $\mathrm{T}$ and $\mathrm{B}$ cells.

Notably, the researchers found that, in all three models, a single injection of an anti-CD40L monoclonal antibody (engineered to be devoid of effector function) given to female NOD or NOD.H-2h4 mice at 4-5 weeks of age - months before the development of overt disease - could prevent the development of pSS, autoimmune thyroiditis and T1DM in aged mice. This result was a surprise because prior studies found that injection of CD40-Fc into the salivary glands of aged mice with overt disease had little effect on disease. Intriguingly, transient CD40L blockade also suppressed the expression of genes related to circadian clock and bone formation pathways, which might have a role in pSS, as well as genes linked to innate immunity and inflammation. The beneficial effect of systemic CD40L blockade, the researchers report explains, does not merely result from local inhibition of autoreactivity in target tissues, but rather corrects the dysregulation of germinal centres in the splenic microenvironment. Of note, the appearance of spontaneous germinal centres in the spleens of untreated NOD.H-2h4 mice coincided with their first oestrus cycle, suggesting that oestrogen may drive the female sex bias in pSS.

Overall, Mahmoud and co-workers show that early dysregulation of immune responses to self-antigens in the spleen is the initial trigger of autoimmunity in NOD and NOD.H-2h4 mice, and that this dysregulation induces (and is then amplified by) the development of tertiary lymphoid structures in target tissues later in life, resulting in local autoantibody production and disease progression.

Although early clinical trials of anti-CD40L therapy were halted owing to thromboembolic complications, a number of new agents engineered to avoid platelet aggregation are currently being investigated in pSS and other diseases.

Caroline Barranco

ORIGINAL ARTICLE Mahmoud, T. I. et al. Autoimmune manifestations in aged mice arise from early-life immune dysregulation. Sci. Transl. Med. http://dx.doi.org/10.1126/scitranslmed. aag0367 (2016) 